Artículo

\title{
Efecto de la oscilación térmica en la calidad y rendimiento de cebolla blanca en el Valle de Culiacán, Sinaloa
}

\author{
Guadalupe Alfonso López-Urquídez ${ }^{1}$ \\ Juan Carlos Cordero-Armenta ${ }^{1}$ \\ Ángel Roberto Martínez-Campos² \\ Jorge Alberto Edeza-Urías ${ }^{1}$ \\ Martín Abraham Tirado-Ramírez ${ }^{1}$ \\ Carlos Alfonso López-Orona ${ }^{1 \S}$
}

${ }^{1}$ Universidad Autónoma de Sinaloa-Facultad de Agronomía. Carretera Culiacán Eldorado km 17.5, Culiacán de Rosales, Sinaloa, México. CP. 80398. (alfonsolopezurquidez@uas.edu.mx; carlos_ cordero1@ hotmail.com; edeza117@gmail.com; tiradoma6@hotmail.com). ${ }^{2}$ Universidad Autónoma del Estado de México-Instituto de Ciencias Agropecuarias y Rurales. El Cerrillo, Piedras Blancas, Toluca, Estado de México, México. CP. 50295. (armartinezc@uaemex.mx).

${ }^{\S}$ Autor para correspondencia: clopezorona@uas.edu.mx.

\section{Resumen}

La cebolla es uno de los cultivos alimenticios más importantes a nivel mundial. Sin embargo, la producción de esta hortaliza es condicionada por factores ambientales como la temperatura. El presente trabajo de investigación realizó en el ciclo otoño-invierno 2016-2017 y tuvo como objetivo estudiar el efecto de la temperatura en el contenido de fibra, proteína, cenizas y ${ }^{\circ}$ Brix en el cultivo de cebolla. Se analizó también la capacidad de este cultivo en la captura de carbono. Con respecto a la oscilación térmica el coeficiente de determinación fue de 0.84; es decir, que esta variable dependiente explica $84 \%$ del comportamiento del peso del bulbo. Asimismo, se encontró que la correlación entre el peso y el diámetro de la cebolla fue de 0.943 , que $94 \%$ del comportamiento de una variable depende de la otra, donde el valor de la pendiente indica que, por cada centímetro de diámetro de la cebolla, esta aumenta $98.6 \mathrm{~g}$ en peso. Con respecto al análisis bromatológico no se encontraron diferencias estadísticas significativas. Asimismo, aunque no hubo diferencias estadísticas significativas entre los tratamientos en referencia a la fijación de bióxido de carbono, si se observó una mayor concentración en el tratamiento que fue el que se desarrolló en condiciones de temperatura más bajas, teniendo un valor de $1.56 \mathrm{CO}_{2}\left(\mathrm{t} \mathrm{CO}_{2}\right.$ eq ha- $\left.{ }^{-1}\right)$.

Palabras clave: bulbificación, captura de carbono, desarrollo vegetal.

Recibido: enero de 2021

Aceptado: marzo de 2021 


\section{Introducción}

La cebolla (Allium cepa L.) es la segunda hortaliza alimenticia más importante a nivel mundial después del tomate con 96773819 t producidas a nivel mundial (FAOSTAT, 2018). En México 2018, se cosecharon 50167.78 ha con una producción de 1572607.99 t y una producción media de $31.35 \mathrm{t} \mathrm{ha}^{-1}$ acorde a datos del Servicio de Información Agroalimentaria y Pesquera (SIAP, 2018). Donde Sinaloa, el mismo año cosechó 1962.85 ha, produciendo 53513.22 t con un rendimiento promedio de $27.26 \mathrm{t} \mathrm{ha}^{-1}$ (SIAP, 2018). Asimismo, la agricultura en Sinaloa ha sufrido grandes daños en los últimos años debido a fenómenos meteorológicos extraordinarios, generados en gran medida por efecto del cambio climático, el cual se debe en parte a las actividades agrícolas que implican la emisión de gases efecto invernadero. Así, unos de los principales factores que influyen en la formación del bulbo de cebolla son: longitud del día, temperatura y variedad del cultivo (Lancaster et al., 1996; Brewster, 2008).

Así mismo, de acuerdo con la FAO, la agricultura puede contribuir a reducir las emisiones de gases de efecto invernadero realizando, entre otras actividades, el uso de variedades de cultivo más eficaces, el manejo del suelo orgánico, la agricultura de conservación y sistemas agroforestales, reducir la emisión de gases de efecto invernadero, pues de acuerdo con esta organización, las tierras de pasto y cultivo bien manejadas pueden secuestrar cantidades significativas de carbono, pues $40 \%$ de la biomasa de la tierra, y con ella el carbono biológico, está directa o indirectamente manejada por agricultores, silvicultores o pastores.

Con base a lo anterior, el objetivo del presente trabajo fue determinar el rendimiento y calidad del bulbo de cebolla blanca después ser establecida en diferentes fechas de siembra y temperaturas, además de determinar el contenido de solidos solubles, proteína, fibra, cenizas y analizar el potencial de captura de carbono del cultivo de cebolla.

\section{Material y métodos}

\section{Localización y condiciones de áreas experimentales}

El estudio se realizó en el ciclo otoño-invierno 2016-2017, bajo condiciones de riego por goteo, en un predio perteneciente a la sindicatura de Quilá, Valle de Culiacán, Sinaloa, situado a $24^{\circ} 27^{\prime}$ 27.64" latitud norte, $107^{\circ} 16^{\prime} 1.45^{\prime}$ ' longitud oeste del meridiano de Greenwich y una altitud de 40 m, el clima de la región es BSo(h,) w (e), descrito como clima seco estepario (B) con un cociente de precipitación entre la temperatura menor que $22.9{ }^{\circ} \mathrm{C}$ (BSo), un régimen térmico muy cálido con una temperatura media anual de $26.8{ }^{\circ} \mathrm{C}$ y la temperatura del mes más frío de $18.6{ }^{\circ} \mathrm{C}$ (h’) donde la precipitación media anual es de $525.8 \mathrm{~mm}$ (García, 1988).

En la Figura 1 se muestra el comportamiento diario de la temperatura promedio máxima, temperatura promedio mínima, temperatura promedio media y la oscilación térmica que ocurre desde que se dio el primer trasplante hasta la cosecha. En todos los casos se observa que al inicio del periodo todos los parámetros térmicos inician con una tendencia a la baja, hasta llegar a la mitad del periodo, donde inicia de nuevo el ascenso. El comportamiento térmico a lo largo del ciclo es importante, porque dependiendo de la fecha de siembra será el efecto que los distintos parámetros térmicos tengan sobre el desarrollo de la cebolla. 


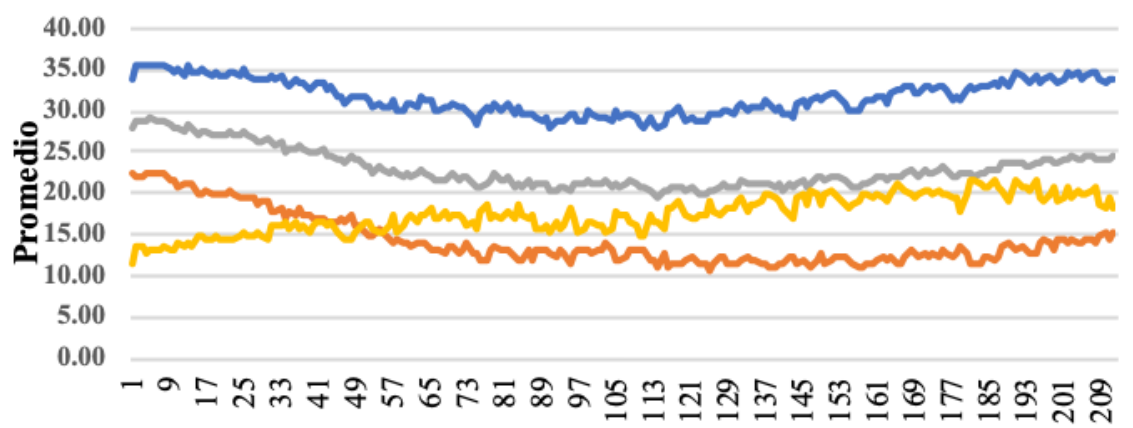

Días de trasplante

\author{
—Tmax - Tmin Tprom Oscilación térmica \\ Nota: Los números del 1 al 211 de esta Figura, corresponden a la fecha de inicio y
}

Figura 1. Comportamiento de la temperatura durante el desarrollo del cultivo de cebolla. Elaboración con datos de la CONAGUA.

\title{
Tipo de suelo
}

Se determinaron algunas características fisicoquímicas del suelo donde se realizó el experimento. La conductividad eléctrica se determinó por el método de extracto de saturación (Aguilar et al., 1987). El pH fue determinado utilizando un potenciómetro (Aguilar et al., 1987). La textura del suelo se calculó con el método de hidrómetro de Bouyoucus (Aguilera y Martínez, 1980). El método para densidad aparente fue por el de probeta. El suelo donde se realizó el estudio fue de textura franco arcillo-arenosa con $33.96 \%$ de arcilla, 6\% de limo y $60.04 \%$ de arena, con un pH 5.7 y una densidad aparte de $1.35 \mathrm{~g} \mathrm{~cm}^{-3}$.

\section{Preparación del suelo, trasplante de plántula y fertirrigación}

El terreno fue preparado mediante un barbecho de $30 \mathrm{~cm}$ de profundidad, posterior a tres rastreos cruzados y se niveló con tablón para romper los terrones de suelo. Posteriormente procedió a formar camas de siembra de $1.6 \mathrm{~m}$ de ancho de centro. En cada cama se formaron seis líneas de plantas con una separación de $12 \mathrm{~cm}$ entre ellas. La distancia entre plantas fue de $12 \mathrm{~cm}$ dispuestas en tresbolillo. El experimento se realizó en condiciones de riego por goteo, utilizando cinta $\left(\right.$ Rivulis $^{\circledR}$ ) con goteros a $20 \mathrm{~cm}$ de separación, $16 \mathrm{~mm}$ de diámetro, espesor de $0.2 \mathrm{~mm}$ y gasto de un litro por hora por gotero.

En cada cama se utilizaron tres cintas. El híbrido de cebolla utilizado fue 'Carta Blanca' $\left(\right.$ Nunhems ${ }^{\circledR}$ ) de días cortos con bulbo de color blanco, forma redonda y madurez fisiológica a los 170-175 días después de la siembra. El cual se produjo la plántula mediante charolas de poliestileno con sustrato Kekkila ${ }^{\circledR}$ (Vantaa, Finland). El trasplante se realizó cuando éstas tenían un grosor de $4 \mathrm{~mm}$ de grosor y altura aproximada de $20 \mathrm{~cm}$ en los nódulos de germinación. Con este método se obtiene cebolla de forma y tamaño uniforme. La fuente de cada nutriente y la dosis de cada uno de ellos se muestran en el (Cuadro 1). 
Cuadro 1. Fuente y dosis de fertilizante ha ${ }^{-1}$ aplicados para el desarrollo de la cebolla.

\begin{tabular}{|c|c|c|c|c|c|c|c|c|c|c|}
\hline Fuente & Formulación & $\begin{array}{l}\text { Fertilizante } \\
\quad(\mathrm{kg})\end{array}$ & $\mathrm{N}$ & $\begin{array}{c}\text { Nitrato } \\
\mathrm{NO}_{3}^{-}\end{array}$ & $\underset{\mathrm{NH}_{4}{ }^{+}}{\text {Amonio }}$ & $\begin{array}{c}\mathrm{P} \\
\mathrm{P}_{2} \mathrm{O}_{5}\end{array}$ & $\mathrm{~K}$ & $\mathrm{Ca}$ & $\mathrm{Mg}$ & $S$ \\
\hline Urea & $46-00-00$ & 20 & 9.2 & 0 & 0 & 0 & 0 & 0 & 0 & 0 \\
\hline $\begin{array}{l}\text { Fosfato } \\
\text { monopotásico }\end{array}$ & $0-52-34$ & 75 & 0 & 0 & 0 & 39 & 25.5 & 0 & 0 & 0 \\
\hline $\begin{array}{l}\text { Nitrato de } \\
\text { potasio }\end{array}$ & $12-00-46$ & 75 & 9 & 9 & 0 & 0 & 34.5 & 0 & 0 & 0.9 \\
\hline $\begin{array}{l}\text { Nitrato de } \\
\text { calcio }\end{array}$ & $\begin{array}{c}15-00-00-30 \\
(\mathrm{CaO})\end{array}$ & 100 & 15 & 15 & 0 & 0 & 0 & 30 & 0 & 0 \\
\hline $\begin{array}{l}\text { Nitrato de } \\
\text { magnesio }\end{array}$ & $\begin{array}{c}11-00-00-16 \\
(\mathrm{Mg})\end{array}$ & 175 & 19.25 & 19.25 & 0 & 0 & 0 & 0 & 28 & 0 \\
\hline $\begin{array}{l}\text { Fosfonitrato } \\
\text { de amonio }\end{array}$ & $33-04-00$ & 450 & 148.5 & 74.25 & 74.25 & 18 & 0 & 0 & 0 & 0 \\
\hline $\begin{array}{l}\text { Sulfato de } \\
\text { amonio }\end{array}$ & $\begin{array}{c}21-0-0-24 \\
(\mathrm{~S})\end{array}$ & 50 & 0 & 0 & 10.5 & 0 & 0 & 0 & 0 & 12 \\
\hline $\begin{array}{l}\text { Ácido } \\
\text { fosfórico }\end{array}$ & $0-52-0$ & 50 & 0 & 0 & 0 & 26 & 0 & 0 & 0 & 0 \\
\hline \multirow{4}{*}{$\begin{array}{l}\text { Sulfato de } \\
\text { potasio }\end{array}$} & $0-0-52-17$ & 75 & 0 & 0 & 0 & 0 & 39 & 0 & 0 & 12.75 \\
\hline & Calculado & & 200.95 & 117.5 & 84.75 & 83 & 99 & 30 & 28 & 25.65 \\
\hline & Requerido & & 200 & 58.47 & 42.17 & 80 & 100 & 30 & 27 & 25 \\
\hline & Diferencia & & 0.95 & 0 & 0 & 3 & -1 & 0 & 1 & 0.65 \\
\hline
\end{tabular}

\section{Medición de la planta de cebolla}

Las plantas de cebolla fueron cosechadas cuando $70 \%$ de éstas en cada uno de los tratamientos presentaron el doblez de la hoja, el cual es estimador que el cultivo ha llegado a madurez fisiológica, por ello se procedió a doblar manualmente al resto de las hojas de las plantas. Dos días después las plantas fueron arrancadas del suelo y colocadas sobre el para completar el sellado del cuello del bulbo. Se midieron las siguientes variables: diámetro ecuatorial del bulbo con vernier (cm) $\left(\right.$ Truper $^{\circledR}$, México), contenido de sólidos solubles $\left({ }^{\circ}\right.$ Brix) mediante refractómetro de mano VEE GEE BX-2 (43003), peso de bulbo (g) mediante balanza analítica (Sartorius ${ }^{\circledR}$ ).

\section{Análisis bromatológicos}

La estimación del carbón fijado se realizó a partir de la composición química proximal de cada estructura. Esta composición química fue determinada mediante análisis bromatológicos especificados en la Asociación Oficial de Análisis Químicos (AOAC, 1999), que incluyeron contenidos de proteína, fibra y cenizas. Para determinar el contenido de proteína se utilizó la metodología de Kjendhal por medio de digestor y destilador Buchi (Caruso et al., 2014).

Los cálculos se realizaron mediante la fórmula $\% \mathrm{~N}=(14.01)$ (ml muestra-ml blanco) $(\mathrm{NHC1}) /($ peso muestra) (10). Donde: 14.01= miliequivalente de nitrógeno; NHC1= normalidad del ácido clorhídrico; ml muestra $=\mathrm{ml}$ de $\mathrm{HCl} 0.1 \mathrm{~N}$ gastados en el destilado de cada muestra; $\mathrm{y}$ $\mathrm{ml}$ blanco $=\mathrm{ml}$ de $\mathrm{HCl} 0.1 \mathrm{~N}$ gastados con el destilado de los blancos. 
La fibra se contabilizó mediante el método de bolsas Ankom aplicando solución de fibra neurodetergente (FND). El cálculo se realizó mediante la fórmula \% FND= $100 \quad\left(\mathrm{~W}_{3^{-}}\right.$ $\left.\left(\mathrm{W}_{1} \mathrm{Xc}\right)\right) / \mathrm{W}_{2}$. Donde: $\mathrm{W} 1=$ peso de la bolsa; $\mathrm{W} 2=$ peso de la muestra; $\mathrm{W} 3=$ peso de la bolsa seca con la fibra después de extracción; y $\mathrm{C}=$ blanco de corrección (peso seco final) dividido entre el peso del blanco inicial. La obtención de cenizas se realizó en crisoles crisol Gooch en Mufla Novatech $^{\circledR}\left(500{ }^{\circ} \mathrm{C}, 3 \mathrm{~h}\right)$ y se tomó referencia del peso final de las cenizas.

\section{Fijación de carbono atmosférico}

Para determinar el porcentaje correspondiente a carbono en cada compuesto, se utilizaron las siguientes fórmulas: \% $\mathrm{C}=\mathrm{PMC} / \mathrm{PMDC} * 100 \quad 1) ; \mathrm{CT}=(\% \mathrm{C}) *(\mathrm{gCM}) / 100) \quad 2) ; \mathrm{EqCO}_{2}=$ $(\mathrm{CT} * 44) / 12$ 3). Donde: $\% \mathrm{C}=$ porcentaje de carbono correspondiente a cada compuesto (24 proteína y 72 para fibra); $\mathrm{PMC}=$ peso molecular del carbono contenido en cada compuesto determinado a partir de la fórmula mínima; $\mathrm{PMDC}=$ peso molecular de la fórmula mínima de cada compuesto (proteína $75 \mathrm{~g} \mathrm{~mol}^{-1}$, fibra $162 \mathrm{~g} \mathrm{~mol}^{-1}$ ); CT= carbono total; $\mathrm{gCM}=$ gramos del compuesto (proteína, fibra) en la muestra; $\mathrm{PS}=$ peso seco de la muestra; $\mathrm{EqCO}_{2}=$ equivalentes de $\mathrm{CO}_{2}$; la determinación del carbono fijado se presenta en toneladas de equivalentes de $\mathrm{CO}_{2}$ por hectárea ( $\mathrm{CO}_{2}$ eq ha ${ }^{-1}$ ) (Pérez et al., 2007).

\section{Diseño experimental y análisis estadísticos}

Se estableció un diseño experimental de bloques completos al azar, evaluando el comportamiento del cultivo de cebolla 'Carta Blanca' sometida a cinco fechas de siembra con temperaturas diferentes, (tratamientos; T1: 07-noviembre; T2: 15-noviembre; T3: 22-noviembre; T4: 29noviembre y T5: 06-diciembre) cada uno con cuatro bloques o repeticiones. Cada bloque constó de una cama de 100 metros de largo, donde en cada una de las camas se distribuyeron los tratamientos al azar mediante el comando= aleatorio. Posteriormente, se ordenó de manera jerárquica cada uno de los números aleatorios por la fórmula= jerarquia(B3, B \$3:B \$7).

Para la selección de la parcela útil (PU) o unidad de muestreo de cada uno de los tratamientos se empleó la fórmula $=$ redondear (aleatorio ()$*(20-1)+1)$. De esta manera la hoja de cálculo proporcionó un número del 1 al 20 para cada tratamiento, el cual representó un metro de largo dentro de la cama del tratamiento la cual fue designada como PU. De cada PU se contaron y midieron los parámetros anteriormente mencionados a todas las plantas de cebolla.

\section{Análisis de datos}

Inicialmente los datos originales de peso de bulbo (g) no representaron los supuestos de normalidad y homocedasticidad, por lo que fueron transformados con la función estándar a partir de raíz cuadrada $(\grave{x}=\sqrt{ } x)$. Donde $\grave{x}=$ valor transformado; $\mathrm{y} x=$ valor original. Esta transformación es utilizada cuando la varianza es proporcional a la media. Además, se invirtieron las medias previamente transformadas a medias de datos originales por la función inversa con $\left(x=\dot{x}^{2}\right)$. Posteriormente a la transformación se cumplieron los supuestos mediante las pruebas de ShapiroWilk y Bartlett, procediendo con Andeva y Tukey $(p \leq 0.05)$. 
Así mismo, se sometieron los datos originales de peso y diámetro de bulbo $(\mathrm{g}),(\mathrm{mm})$ al análisis de correlación y regresión lineal simple. Se utilizó estadística no paramétrica Kruskall-Wallis y comparación de medianas de Dunn $(p \leq 0.05)$ con los datos originales de sólidos solubles $\left({ }^{\circ} \mathrm{Brix}\right)$, debido a que éstos no cumplieron con los supuestos anteriormente mencionados por la vía de original y la transformación de datos. Para los datos provenientes de análisis bromatológicos y fijación de $\mathrm{CO}_{2}$ atmosférico se analizaron los supuestos de normalidad y homocedasticidad para posterior realización del Andeva y prueba de comparación de medias Tukey $(p \leq 0.05)$. Por otra parte, con los datos de peso y diámetro de la cebolla se realizó un análisis de correlación observando que existe una relación entre dichas variables. El análisis de regresión lineal se dio a partir de una ecuación de la relación entre una variable independiente y otra dependiente. Los análisis se realizaron con el paquete estadístico Xlstat (Addinsoft, 2019).

\section{Resultados y discusión}

\section{Peso del bulbo}

El rendimiento fue representado por el peso del bulbo de la cebolla, el cual presentó diferencia estadística significativa $(p \leq 0.05)$. Encontrando que las cebollas que se trasplantaron el 06 de diciembre (fecha tardía) tuvieron en promedio menor desarrollo $\mathrm{T} 5=153.19 \mathrm{~g}$, mientras las sembradas (fecha temprana) presentaron mayor peso en bulbo $\mathrm{T} 1=348.62 \mathrm{~g}$ (Cuadro 2).

Cuadro 2. Rendimiento bulbar de cebolla sometida a diferentes fechas de siembra.

\begin{tabular}{ccc}
\hline Tratamiento & Fecha de siembra & Peso $(\mathrm{g})$ \\
\hline T1 & 07 noviembre & $34862 \mathrm{a}$ \\
T2 & 15 noviembre & $29287 \mathrm{~b}$ \\
T3 & 22 noviembre & $33859 \mathrm{ab}$ \\
T4 & 29 noviembre & $19387 \mathrm{c}$ \\
T5 & 06 diciembre & $15319 \mathrm{c}$ \\
\hline
\end{tabular}

Promedios seguidos de letras comunes indican diferencias significativas entre tratamientos (Tukey; $p \leq 0.5$ ).

De acuerdo con McClung y Davis (2010) la temperatura es un factor determinante en el desarrollo de las plantas, lo cual incluye rangos en donde la planta no se encuentra estresada. Partiendo de ese criterio, en esta investigación se relaciona el peso de la cebolla con diferentes parámetros de la temperatura, encontrando que hay algunos que si influyen en los resultados obtenidos. El comportamiento de la temperatura durante el desarrollo del experimento se muestra en la (Figura 2). En el tercer tratamiento se presentaron las temperaturas promedio máximas más bajas con $30.03{ }^{\circ} \mathrm{C}$, mientras que la más baja ocurrió en la tercera fecha.

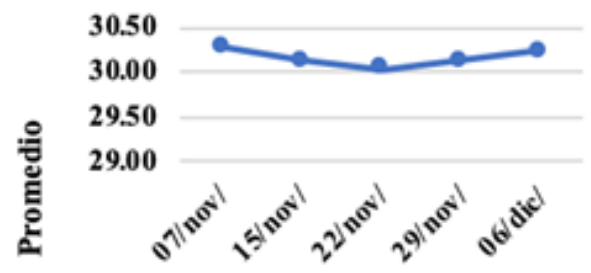

Fecha

Figura 2. Temperatura máxima promedio en el desarrollo del cultivo de cebolla (CONAGUA, 2015). 
Por otra parte, en la Figura 3, se muestra que las temperaturas mínimas descendieron de acuerdo con la fecha de los tratamientos, encontrando que para el tratamiento 1, la temperatura mínima promedio fue de $13.06{ }^{\circ} \mathrm{C}$, mientras que para el tratamiento cinco fue $12.27^{\circ} \mathrm{C}$. Con respecto a la Figura 4, los datos muestran que la oscilación térmica para cada tratamiento fue diferente, encontrando que primero tuvo los valores más bajos, presentándose una tendencia a incrementar la oscilación térmica para cada tratamiento, de acuerdo con la fecha de trasplante.

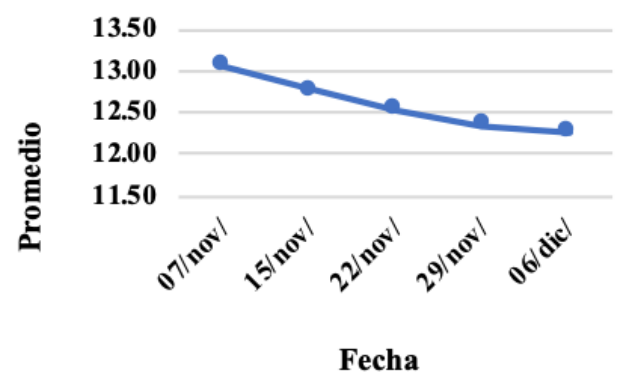

Figura 3. Temperatura mínima promedio en el desarrollo del cultivo de cebolla. Elaboración con datos de la CONAGUA (2015).

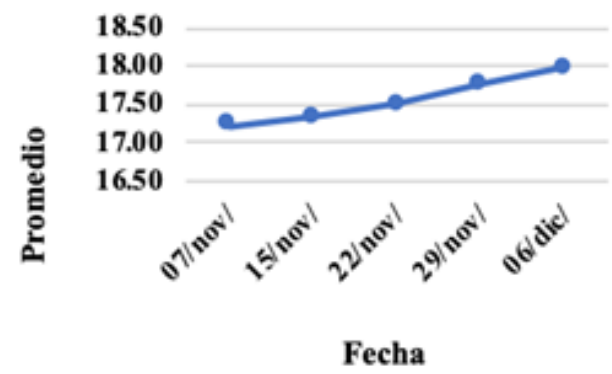

Figura 4. Oscilación térmica promedio de la temperatura en el desarrollo del cultivo de cebolla. Elaboración con datos de la CONAGUA (2015).

Se analizaron los datos de los parámetros de temperatura: temperatura máxima promedio, temperatura mínima promedio, promedio de temperatura y oscilación térmica. Al hacer el análisis de regresión lineal, se encontró que con los cuatro parámetros se tiene significancia estadística. Sin embargo, los valores del coeficiente de determinación son más altos en los parámetros de oscilación térmica y la variación de las temperaturas mínimas promedio.

Con respecto a la oscilación térmica el coeficiente de determinación fue de 0.84 , es decir, que esta variable dependiente explica $84 \%$ del comportamiento del peso del bulbo. Al respecto Steer (1980) encontró que el desarrollo del bulbo era más lento cuando la diferencia entre la temperatura nocturna era de $15{ }^{\circ} \mathrm{C}$ que cuando la oscilación era de $5{ }^{\circ} \mathrm{C}$. Una de las razones de ello puede ser que la cebolla es muy sensible a la variación diaria de la temperatura, pues durante las primeras horas de la mañana la planta se encuentra erguida, mientras que al medio día, durante las temperaturas máximas, la planta dobla sus hojas, lo que puede implicar pérdida de energía, luego entonces, entre más alta sea la oscilación térmica, mayor será el movimiento que tengan las hojas (Figura 5). 


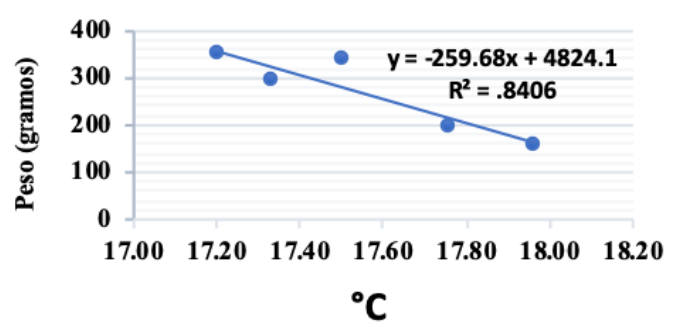

Figura 5. Influencia de la oscilación térmica en el rendimiento de la cebolla en Sinaloa. Elaboración con datos de la temperatura de la CONAGUA (2018).

Las plantas que se desarrollaron bajo temperaturas máximas más altas tuvieron un menor desarrollo, debido a que las altas temperaturas acortaron el ciclo de vida, al respecto Lescay y Moya (2006) encontraron que el rendimiento de la cebolla disminuye al aumentar la temperatura. Por otra parte, algunas especies de plantas ajustan el ángulo de la hoja con relación a los rayos solares al medio día cuando se tiene el mayor calentamiento (Jones y Rotenberg, 2001). La planta realiza esta acción para protegerse contra el estrés hídrico. Sin embargo, al reducir la intercepción de la luz, afecta la capacidad de mantener altas tasas de asimilación de $\mathrm{CO}_{2}$ durante este periodo en plantas bien regadas (Pastenes et al., 2004). Esto afecta entonces la actividad fotosintética y con ello el desarrollo de la planta de cebolla.

El otro parámetro de la temperatura con un coeficiente de determinación alto fue la variación de la temperatura mínima. Se observa que conforme aumentan los valores de temperatura mínima, se tiene también un incremento de los rendimientos.

Esto puede estar relacionado con el hecho de que las cebollas trasplantadas más temprano tuvieron un mejor desarrollo durante el periodo antes de la bulbificación, pues estaban más alejadas de la temperatura mínima para el desarrollo de las hojas que es de 6 a $7{ }^{\circ} \mathrm{C}$ según Kalbarczyk y Kakbarczyc (2015); es decir, las plantas tenían unas condiciones más favorables para el desarrollo de la hoja. En este aspecto Lancaser et al. (1996) señala que en el estudio que ellos realizaron, el crecimiento del bulbo estuvo relacionado con el tiempo térmico acumulado antes de la bulbificación. Por el contrario, en las cebollas que se trasplantaron en fechas más tardías esta etapa del desarrollo ocurrió cuando las temperaturas estaban más cercanas al punto crítico de desarrollo.

Las cebollas trasplantadas durante las primeras fechas tuvieron un desarrollo prebulbificación adecuado, acumulando nutrientes en sus hojas. Esto permite que las cebollas estén en condiciones favorables cuando ellas capten la disminución de la temperatura, que indica que la planta debe entrar en un periodo de dormancia para soportar el frío.

Ante esta situación la planta inicia el proceso de bulbificación. Al respecto Thomashow (1999) indica que la aclimatación al frio implica muchos cambios bioquímicos y fisiológicos incluidos la modificación de la composición de la membrana, aumento en el contenido de proteínas solubles, el incremento de los niveles de proteínas y azúcares que le permiten a la planta resistir la congelación. Por el contrario, las plantas que se sembraron más tarde reciben dicha señal cuando aún no han acumulado nutrientes suficientes en sus hojas, por lo que el proceso de bulbificación es deficiente. 
La formación adecuada de hojas antes y durante la bulbificación es muy importante, dado que la translocación de nutrientes de la hoja verde al bulbo es determinante en el peso de la cebolla (López et al., 2017). Aunado a ello en la última etapa de la formación del bulbo, la planta se encuentra bajo condiciones de altas temperaturas, lo cual acelera el proceso de maduración pues, la acumulación de calor se realiza en menos tiempo que las cebollas trasplantadas más temprano. Al respecto, Ikeda et al. (2019) señalan que en el estudio que ellos realizaron, el crecimiento del bulbo se detuvo a temperaturas relativamente altas.

En el crecimiento del bulbo también influye el fotoperiodo y podría considerarse que tiene un efecto que se combina con la temperatura (Chope et al., 2012). En este caso es complicado estimar cuál es el factor determinante, pues el lugar donde se desarrolló este experimento, la temperatura es altamente dependiente de la duración del día. Al respecto Brewster (2008) considera que la influencia de estos dos factores dependerá de las variedades de cebolla que se encuentren en el mercado.

Las diferencias de peso encontradas entre los tratamientos, también puede aplicar para el diámetro de la cebolla, pues al realizar el análisis de correlación entre el peso y el diámetro de la cebolla se observó que sí había significancia estadística en la relación, como se puede observar en el apartado siguiente.

\section{Relación diámetro y peso de la cebolla}

Al realizar el análisis de varianza de correlación entre peso y diámetro de la cebolla, se observó que sí había significancia estadística en la regresión con un valor crítico de F de 9.169E-144. Asimismo, se encontró que la correlación entre el peso y el diámetro de la cebolla fue de 0.943; es decir, que $94 \%$ del comportamiento de una variable depende de la otra. Además, el valor de la pendiente (b) indica que por cada centímetro que, del diámetro de la cebolla, esta aumenta $98.6 \mathrm{~g}$ en peso como se observa en la (Figura 6).

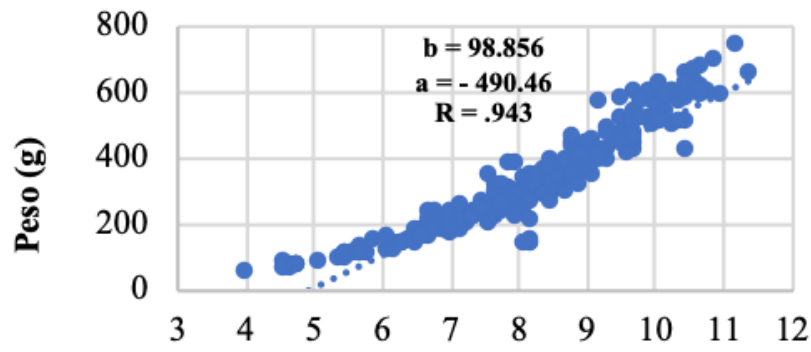

Diámetro (cm)

Figura 6. Relación entre peso y diámetro de la cebolla. Grados Brix en el bulbo de la cebolla.

\section{Grados Brix en el bulbo de la cebolla}

La prueba estadística no paramétrica de Kruskal-Wallis aplicada para analizar la diferencia entre los tratamientos con respecto al contenido de ${ }^{\circ}$ Brix, indica que se tuvo una diferencia estadística significativa. 
A partir de lo anterior se realizó una comparación de rangos mediante el procedimiento de Dunn, encontrando que la menor concentración de ${ }^{\circ}$ Brix se obtuvo en el tratamiento cinco, el cual se compone de las cebollas que se trasplantaron más tarde. Por el contrario, la que tuvo mayor concentración de ${ }^{\circ}$ Brix fue sembrada en fechas intermedias.

Las plantas con menor concentración de ${ }^{\circ}$ Brix, son las que se trasplantaron en las últimas fechas, razón por la cual en la última etapa de su desarrollo ocurrieron las temperaturas más altas. Por el contrario, el tratamiento de fecha intermedia se desarrolló bajo condiciones de temperaturas más bajas (Cuadro 3). Asimismo, tuvo los valores más altos de ${ }^{\circ}$ Brix, esto puede deberse a que la cebolla como otras especies de plantas reacciona a las bajas temperaturas, concentrando azúcares como un mecanismo de defensa para aclimatarse a temperaturas que pueden llegar al punto de congelación (Thomashow, 1999).

Cuadro 3. Comparaciones múltiples por pares mediante el procedimiento de Dunn-prueba bilateral.

\begin{tabular}{ccccc}
\hline Muestra & Frecuencia & Suma de rangos & Media de rangos & Grupos \\
\hline 07 noviembre & 75 & 14441 & 192.55 & $\mathrm{C}$ \\
15 noviembre & 78 & 14683.5 & 188.25 & $\mathrm{C}$ \\
22 noviembre & 58 & 14353 & 247.47 & $\mathrm{D}$ \\
29 noviembre & 59 & 6642.5 & 112.59 & $\mathrm{~B}$ \\
06 diciembre & 57 & 3508 & 61.54 & $\mathrm{~A}$ \\
\hline
\end{tabular}

Se encontró que conforme aumenta la temperatura máxima, disminuye la concentración de ${ }^{\circ}$ Brix, encontrando que la variable independiente, determina el comportamiento del rendimiento en $24 \%$ y que por cada $0.1{ }^{\circ} \mathrm{C}$ que aumenta la temperatura máxima, la concentración disminuye $36^{\circ}$ Brix.

\section{Constante térmica}

El desarrollo de la cebolla fue afectado por la velocidad de acumulación de las unidades calor, encontrando que una de las consecuencias del incremento de la temperatura durante el periodo de desarrollo entre el trasplante y la flexión de la hoja, fue más corto conforme la cebolla se trasplantó más tarde. Las cebollas trasplantadas en la primera fecha acumularon una constante térmica de $2621.88{ }^{\circ} \mathrm{C}$, tuvieron un periodo de desarrollo que duró 120 días, mientras que los tratamientos donde las cebollas se trasplantaron más tarde tuvieron un periodo de 110 días, acumulando un promedio de $2359.08^{\circ} \mathrm{C}$.

Una de las razones por las cuales el periodo de desarrollo de la cebolla fue más corto en los tratamientos trasplantados más tarde puede deberse a que las temperaturas se incrementaron al final del ciclo vegetativo, con lo cual se aceleró la maduración de la planta.

El tratamiento que se trasplantó primero presenta los valores de temperatura más altos, debido a que, en las primeras etapas de desarrollo de la planta, la temperatura fue más alta que el resto de los tratamientos, lo que pudo afectarla en su desarrollo al durar más tiempo con temperaturas cálidas antes de llegar a la bulbificación. Esto sugiere que la constante térmica influye de manera diferenciada en las distintas etapas de desarrollo de la cebolla (Cuadro 4). 
Cuadro 4. Fecha de siembra, días a cosecha y temperatura acumulada para la cebolla trasplantada cada semana.

\begin{tabular}{cccccc}
\hline \multirow{2}{*}{ Fecha de trasplante } & Días a cosecha & Máxima & Mínima & Promedio & Oscilación térmica \\
\cline { 2 - 6 } & \multicolumn{5}{c}{ Temperatura acumulada $\left({ }^{\circ} \mathrm{C}\right)$} \\
\hline 07 noviembre & 120 & 3663.01 & 1580.75 & 2621.88 & 2082.26 \\
15 noviembre & 115 & 3493.46 & 1482.86 & 2488.16 & 2010.6 \\
22 noviembre & 110 & 3333.72 & 1390.74 & 2362.23 & 1942.98 \\
29 noviembre & 110 & 3342.93 & 1371.18 & 2357.05 & 1971.74 \\
06 diciembre & 110 & 3356.13 & 1362.03 & 2359.08 & 1994.1 \\
\hline
\end{tabular}

\section{Análisis bromatológico}

A pesar de que no se encontraron diferencias estadísticas significativas con respecto al análisis bromatológico, sí se observa que el tratamiento que se desarrolló bajo las temperaturas más bajas tuvo la mayor concentración de proteínas, lo cual también puede estar relacionado con la reacción que tienen algunas especies vegetales de concentrar proteínas en sus células como un mecanismo de defensa contra las bajas temperaturas. Este proceso puede contribuir al aumento de materia seca en la cebolla y por lo tanto en el rendimiento.

Los resultados de los análisis bromatológicos en cuanto a fibra, proteína y cenizas Cuadro 5 no se encontró diferencia significativa en ninguno de los casos y se puede observar que la cantidad es muy similar en cada una de ellas, eso quiere decir que, aunque se modifique la fecha y densidad en el cultivo, este no modificará su composición por lo menos en cuanto a las características que se evaluaron.

Cuadro 5. Resultados de análisis bromatológicos

\begin{tabular}{cccc}
\hline Tratamiento & Fibra & Proteína & Ceniza \\
\hline 07 noviembre & $5.86 \mathrm{a}$ & $7.69 \mathrm{a}$ & $5.92 \mathrm{a}$ \\
15 noviembre & $6.16 \mathrm{a}$ & $8.95 \mathrm{a}$ & $5.44 \mathrm{a}$ \\
22 noviembre & $6.09 \mathrm{a}$ & $9.53 \mathrm{a}$ & $5.87 \mathrm{a}$ \\
29 noviembre & $6.57 \mathrm{a}$ & $8.35 \mathrm{a}$ & $5.77 \mathrm{a}$ \\
06 diciembre & $6.23 \mathrm{a}$ & $6.85 \mathrm{a}$ & $5.26 \mathrm{a}$ \\
\hline
\end{tabular}

Valores con la misma letra son estadísticamente iguales, significancia de 0.05 .

Después de aplicar la fórmula para cada compuesto, se determinó la cantidad de $\mathrm{CO}_{2}$ fijado por hectárea de cada uno de los tratamientos, encontrando que son estadísticamente similares, según lo reportado en una investigación realizada en Zinacantepec estado de México por Paulino (2013), la cantidad de $\mathrm{CO}_{2}$ fijado en el cultivo de cebolla es superior al carbono fijado de pino en los bosques que corresponde al $0.847 \mathrm{t} \mathrm{ha}^{-1}$ al año, esto da una idea del potencial del cultivo como fijador de carbono, no obstante el mismo autor reportó que el bosque tropical lluvioso fijaba $32.34 \mathrm{t} \mathrm{CO}_{2}$ año $^{-1}$ el cultivo de papa $30.08 \mathrm{t} \mathrm{CO}_{2} \mathrm{año}^{-1}$, siendo estos muy superiores en cuanto a captura de carbono comparados con el cultivo de cebolla. 
Aun cuando no hubo diferencias estadísticas significativas entre los tratamientos (Cuadro 6) en referencia a la fijación de bióxido de carbono, sí se observa una mayor concentración en el tratamiento tres que fue el que se desarrolló en condiciones de temperatura más bajas, teniendo un valor de $1.56 \mathrm{CO}_{2}\left(\mathrm{t} \mathrm{CO}_{2} \mathrm{eq} \mathrm{ha}^{-1}\right)$. Lo cual también puede estar relacionado con las bajas temperaturas bajo las cuales se desarrolló el tercer tratamiento. El incremento de $\mathrm{CO}_{2}$ puede ser una consecuencia indirecta de las bajas temperaturas que a su vez provocaron el incremento de proteínas y azúcares y que como consecuencia de ello se tuvo un incremento de carbono.

Cuadro 6. Equivalente de fijación de $\mathrm{CO}_{2}$ total, de la planta.

\begin{tabular}{cc}
\hline Tratamiento & $\mathrm{CO}_{2}\left(\mathrm{t} \mathrm{CO}_{2} \mathrm{eq} \mathrm{ha}^{-1}\right)$ \\
\hline 07 noviembre & $1.28 \mathrm{a}$ \\
15 noviembre & $1.43 \mathrm{a}$ \\
22 noviembre & $1.56 \mathrm{a}$ \\
29 noviembre & $1.52 \mathrm{a}$ \\
06 diciembre & $1.44 \mathrm{a}$ \\
\hline
\end{tabular}

Valores con la misma letra son estadísticamente iguales, significancia de 0.05 .

\section{Conclusiones}

El diámetro y peso de la cebolla están altamente correlacionados, por lo que, al conocer el diámetro del bulbo en el campo, se puede tener una estimación del rendimiento esperado en un predio. Las plantas de cebolla que se desarrollaron bajo condiciones de temperaturas más bajas tuvieron una mayor concentración de grados Brix, lo cual puede deberse a la cebolla como otras especies de plantas reacciona a las bajas temperaturas, concentrando azúcares como un mecanismo de defensa para aclimatarse a temperaturas bajas, lo cual le permite soportar temperaturas que lleguen al punto de congelación.

Las plantas que se trasplantaron en más tarde tuvieron un periodo de vida más corto, lo cual puede deberse a que las temperaturas se incrementaron al final del ciclo vegetativo, con lo cual se aceleró la maduración de la planta. Los resultados de los análisis bromatológicos en cuanto a fibra, proteína y cenizas no muestran diferencia significativa en ninguno de los casos y se puede observar que la cantidad es muy similar en cada una de ellas, eso quiere decir que, aunque se modifique la fecha y densidad en el cultivo, este no modificará su composición por lo menos en cuanto a las características que se evaluaron.

\section{Literatura citada}

Addinsoft. 2019. XLSTAT statistical and data analysis solution. Boston, USA. https://www.xlstat.com.

Aguilar, S. A.; Etchevers, J. D. y Castellanos, J. Z. 1987. Análisis químico para evaluar la fertilidad del suelo. Publicación Especial 1. Sociedad Mexicana de la Ciencia del Suelo. México. (Ed.). 217 p.

Aguilera, M. y Martínez, R. 1980. Relaciones agua-suelo-planta-atmósfera. Universidad Autónoma de Chapingo (UACH). Texcoco, Estado de México. 86 p.

AOAC. 1999. Official Methods of Analysis, 16 ${ }^{\text {th }}$. Ed. AOAC International, MD, EE. UU. 1141 p. 
Brewster, J. L. 2008. Onions and Other Vegetable Alliums. 2nd Ed. In: Crop Production Science in Horticulture. Horticulture Research International. Wellesbourne, UK. 432 p.

Caruso, G.; Conti, S.; Villari, G.; Borrelli, C.; Melchionna, G.; Minutolo, M.; Russo, G. and Amalfitano, C. 2014. Effects of transplanting time and plant density on yield, quality and antioxidant content of onion (Allium cepa L.) in southern Italy. Sci. Hortic. 166(1):111120. https://doi.org/10.1016/j.scienta.2013.12.019.

Chope, G. A.; Cools, K.; Hammond, J. P.; Thompson, A. J. and Terry, L. A. 2012. Physiological, biochemical and transcriptional analysis of onion bulbs during storage. Annals Bot. 109(4):819-83.

FAOSTAT. 2018. Production statistics of crops. http://www.fao.org/faostat/es/\#data/QC/ visualize.

García, E. 1988. Modificaciones al sistema de clasificación climática de Köppen. Para adaptarlo a las condiciones de la República Mexicana. $4^{\mathrm{a}}$ (Ed.). Offset Larios. México, DF. 90 p.

Ikeda, H.; Kinoshita, T.; Yamamoto, T. and Yamasaki, A. 2019. Sowing time and temperature influence bulb development in spring-sown onion (Allium cepa L.). Sci. Hortic. 244(1):242-248. https://doi.org/10.1016/j.scienta.2018.09.050.

Jones, H. G.; and Rotenberg, E. 2001. Energy, Radiation and Temperature Regulation in Plants. In: eLS. John Wiley and Sons, Ltd. Chichester, UK. 1-9 pp. https://doi.org/10.1002/ 9780470015902.A0003199.PUB2.

Kalbarczyk, R. and Kalbarczyk, E. 2015. The adverse effect of the long-term trend of the air temperature in Poland on the yield of onion Niekorzystny przebieg wieloletniej temperaturypowietrza w Polsce ograniczającej plon ogólny cebuli (Allium cepa L). J. Central Eur. Agric. 16(1):41-53. Doi: 10.5513/JCEA01/16.1.1538.

Lancaster, J. E.; Triggs, C. M.; De Ruiter, J. M. and Gandar, P. W. 1996. Bulbing in onions: photoperiod and temperature requirements and prediction of bulb size and maturity. Annals Bot. 78(4):423-430. https://doi.org/10.1006/anbo.1996.0138.

Lescay, E. y Moya, C. 2006. Influencia de los factores climáticos sobre algunas variables morfoagronómicas en la producción de bulbos de cebolla (Allium cepa, L.) en la región oriental de cuba. Cultivos Tropicales. 27(4):73-75. http://www.redalyc.org/articulo.oa? $\mathrm{id}=193215912013$.

López, U. G.; Gastélum González, S.; Díaz Valdés, T.; Ayala Tafoya, F.; Madueño Martínez, J. y López Orona, C. A. 2017. Incremento del tamaño y peso del bulbo de cebolla (Allium cepa L.) por translocación de nutrientes. Rev. Mex. Cienc. Agríc. 8(7):1647-1652. https://doi.org/https://doi.org/10.29312/remexca.v8i7.519.

McClung, C. R. and Davis, S. J. 2010. Ambient thermometers in plants: from physiological outputs towards mechanisms of thermal sensing. Current Biol. 20(24):1086-1092. DOI: https://doi.org/10.1016/j.cub.2010.10.035.

Pastenes, C.; Pimentel, P. and Lillo, J. 2004. Leaf movements and photoinhibition in relation to water stress in field-grown beans. J. Exp. Bot. 56(411):425-433. doi:10.1093/jxb/eri061.

Paulino-Flores, M. 2013. Evaluación de la captura de carbono en tres sistemas de producción de papa en Zinacantepec, Estado de México. Tesis de licenciatura. Universidad Autónoma del Estado de México. Toluca, Estado de México. 58 p. http://ri.uaemex.mx/handle/ 20.500.11799/58604.

Pérez, C. F. J.; Carballo, C. A.; Santacruz, V. A.; Hernández, L. A. y Molina M. J. C. 2007. Calidad fisiológica en semillas de maíz con diferencias estructurales. Agric. Téc. Méx. 33(1):53-61. 
SIAP. 2018. Anuario Estadístico de la Producción Agrícola. http://infosiap.siap.gob.mx/ aagricola_siap_gb/icultivo/index.jsp.

Steer, B. T. 1980. The role of night temperature in the bulbing of onion (Allium cepa L.) J. Agric. Res. 31(3):519-523. https://doi.org/10.1071/AR9800519.

Thomashow, M. F. 1999. Plant cold acclimation: freezing tolerance genes and regulatory mechanisms. Annu. Rev. Plant Physiol. Plant Mol. Biol. 50(1):571-599. Doi: 10.1146/annurev.arplant.50.1.571. 